\title{
OntoLO: Um modelo ontológico para avaliar o desempenho acadêmico na Educação a Distância
}

\author{
Laécio A. Costa ${ }^{1,2}$, Leandro P. Sanches ${ }^{2}$, Laís N. Salvador ${ }^{2}$, Marlo V. S. \\ Souza $^{2}$, Ricardo R. Amorim ${ }^{3}$ \\ ${ }^{1}$ Coordenação de Informática - Instituto Federal do Sertão Pernambucano \\ (IFSertão) - Petrolina, PE \\ ${ }^{2}$ Programa de Pós-Graduação em Ciência da Computação - Universidade \\ Federal da Bahia (UFBA) - Salvador, BA \\ ${ }^{3}$ Departamento de Educação - Universidade Estadual da Bahia (UNEB) \\ Senhor do Bonfim, BA \\ laecio.costalifsertao-pe.edu.br, \\ \{leandrompsanches, amorim.ricardo\}@gmail.com, \{laisns, msouzal\}@ufba.br
}

\begin{abstract}
This paper presents a work in progress that describes an ontology, OntoLO, that aims to formalize Taxonomies of Educational Objectives. The objective of this ontology is to assist in the process of evaluating student academic performance based on these taxonomies. The current stage of this work formalizes two taxonomies referenced and consolidated by theories of learning. With OntoLO it will be possible to make inferences about the learning status of the student, based on the interactions that took place in the Learning Management Systems.
\end{abstract}

Resumo. Este artigo apresenta um trabalho em andamento que descreve uma ontologia, OntoLO, que visa formalizar as Taxonomias de Objetivos Educacionais. $O$ objetivo desta ontologia é auxiliar no processo de avaliação do desempenho acadêmico dos alunos com base nessas taxonomias. $O$ atual estágio deste trabalho formaliza duas taxonomias referenciadas $e$ consolidadas pelas teorias da aprendizagem. Com OntoLO será possível fazer inferências sobre o status de aprendizagem do aluno, com base nas interações que ocorreram nos Sistemas de Gestão de Aprendizagem.

\section{Introdução}

Objetivos Educacionais (OE) são formulações explícitas de uso pedagógico que estão atreladas ao processo de aprendizagem. Para Bloom (1956), os OE são mudanças que ocorrem, ou espera-se ocorrer, nos alunos mediante processo educacional. Ng (2005) define OE como uma declaração da capacidade que pode ser observada como resultado da experiência educacional a partir de um planejamento pré-definido. Esse objetivo é composto por "comportamento para a aprendizagem", "contexto da aprendizagem", "público-alvo" e "nível de desempenho". OE norteiam educadores na construção do caminho pedagógico a ser percorrido pelo aluno que, através de uma estrutura hierárquica, determina níveis de conhecimento a serem adquiridos. Os educadores utilizam verbos que representam os objetivos planejados a serem cumpridos pelos 
VII Congresso Brasileiro de Informática na Educação (CBIE 2018)

Anais do XXIX Simpósio Brasileiro de Informática na Educação (SBIE 2018)

alunos. Através da avaliação da realização desses objetivos, é possível acompanhar a aquisição da habilidade ou competência durante a experiência educacional.

As interações dos alunos representam as experiências acadêmicas realizadas em uma sala de aula virtual, e produzem um grande volume de dados. Com a necessidade de processar e analisar esses dados, surgiu o termo Learning Analytics - LA, que tem como proposta coletar, mensurar, analisar dados e apresentar conhecimento útil sobre a aprendizagem de cada estudante [Long e Siemens, 2011].

A utilização de técnicas de LA e Ontologias Computacionais (OC) que, formalizam uma Taxonomia dos Objetivos Educacionais, auxiliam no processo de avaliação do desempenho acadêmico do estudante. Resultados do mapeamento sistemático realizados por Costa et al. (2018), mostram dois trabalhos [Nussbaumer et al., 2012 e Yago et al., 2018] que utilizam ontologias e formalizam a Taxonomia de Bloom para avaliar o contexto dos Objetos de Aprendizagem (OA), porém não foram identificados estudos que apresentam o uso coordenado de Ontologias e LA para avaliar o desempenho acadêmico norteado por uma estrutura de classificação dos OE.

$\mathrm{O}$ uso de $\mathrm{OC}$ foi motivado pelo fato de possibilitar a conceitualização de um modelo taxonômico abstrato, que seja entendido por agentes de software a fim de auxiliar na análise das interações do aluno em Sistemas de Gestão da Aprendizagem (SGA). Desta forma, esse trabalho apresenta uma proposta de formalização da Taxonomia dos Objetivos Educacionais por meio de OC que, implementada em uma arquitetura de software educacional e aliada a técnicas de LA, auxiliará na análise do desempenho acadêmico dos estudantes da Educação a Distância.

\section{Trabalhos Relacionados}

A ontologia proposta por Lima et al. (2017) objetiva gerenciar OA e suas relações com os OE norteados pela Taxonomia de Bloom. Os autores (ibid) utilizam Mapa de Conteúdos e Mapa de Dependências para representar o conteúdo mais significativo em um determinado OE. Kalou et al. (2012) propuseram um modelo que visa classificar os resultados da aprendizagem alinhados com os Objetivos Educacionais explorando os aspectos do conhecimento baseada na Taxonomia Revisada de Bloom [Anderson e Krathwohl, 2001]. Ng (2005) apresenta uma ontologia para representação dos OE baseado na recomendação de OA de acordo com as experiências dos alunos. Esse modelo tem como objetivo melhorar as regras de sequenciamento dos conteúdos e a disponibilidade de OA para a personalização dos respectivos OE com base na Taxonomia de Bloom. O trabalho de Nussbaumer et al. (2012) apresenta um modelo para auxiliar no monitoramento de ações não observáveis das atividades cognitivas e metacognitivas dos alunos aliada a taxonomia das atividades de aprendizagem. Yago et al. (2018) apresentam uma rede de ontologias para supervisionar a aprendizagem do aluno e recomendar atividades baseado na Taxonomia de Bloom.

As propostas anteriores possuem elementos em comum, como exemplo, a relação de utilização de uma Taxonomia dos OE e OA. Diferentemente dos trabalhos relacionados, essa proposta visa auxiliar o educador no processo de avaliação do desempenho acadêmico através da formalização das taxonomias dos $\mathrm{OE}$ mais referenciadas (Bloom e Bloom Revisada), e das interações dos alunos no SGA. Para isso, foram criadas regras de Lógica de Descrição que possibilitam a inferência sobre 
VII Congresso Brasileiro de Informática na Educação (CBIE 2018)

Anais do XXIX Simpósio Brasileiro de Informática na Educação (SBIE 2018)

questões relacionadas ao desempenho do estudante em uma específica estrutura taxonômica.

\section{Modelo Ontológico - OntoLO}

A ontologia OntoLO (Learning Objectives Ontology), é composta por cinco classes representadas na Figura 1(a). Estas classes foram criadas com base nas duas Taxonomias dos Objetivos Educacionais mais referenciadas (Bloom e Bloom Revisada) e nos modelos ontológicos anteriormente apresentados na Seção 2. A ontologia proposta formaliza essas taxonomias possibilitando a criação de uma versão inicial de uma base de conhecimento em diferentes estruturas de classificação dos Objetivos Educacionais. A Figura 1(b) mostra uma visualização da ontologia através do plugin $\mathrm{VOWL}^{1}$, onde as classes são representadas pelos círculos e os retângulos representam as relações do tipo ObjectProperties. O círculo em destaque vermelho, trata da relação entre a classe Estrutura_classificacao (com instancias das taxonomias) com a união dos indivíduos das classes Desempenho_academico e Objetivo_educacional.

(a)

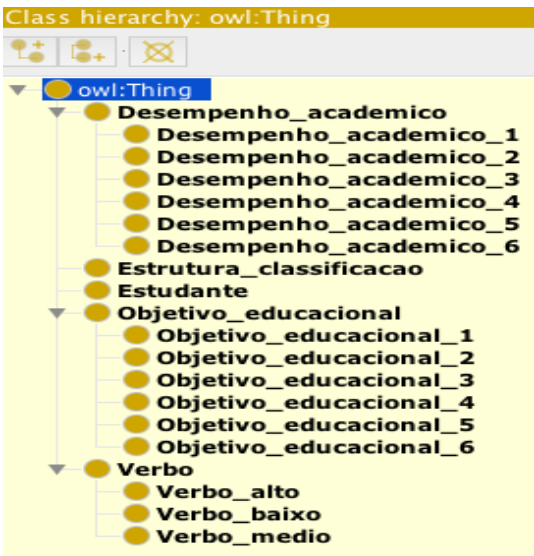

(b)

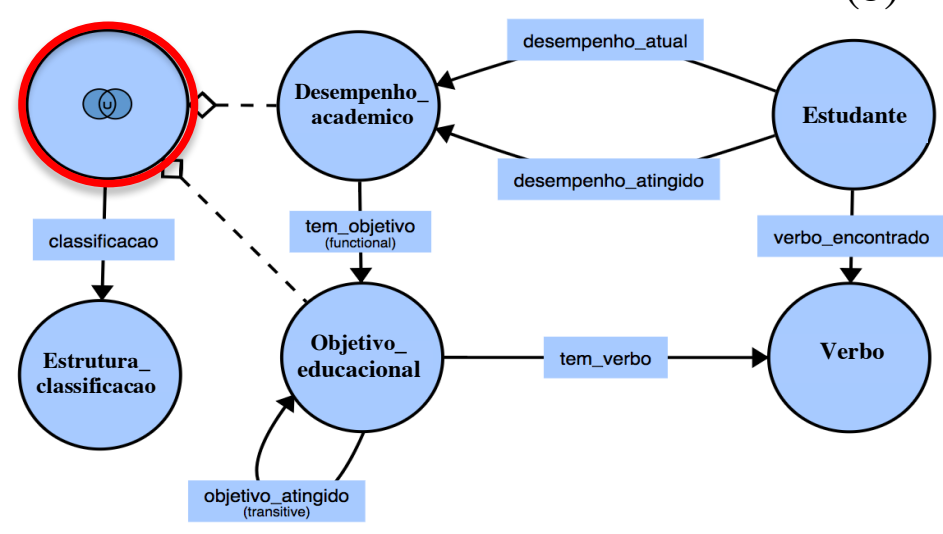

Figura 1: Estrutura da Ontologia OntoLo.

A classe Estrutura_classificação possui instâncias que representam as duas taxonomias (Bloom e Bloom Revisada). A relação classificação associa indivíduos das classes Desempenho_academico e Objetivo_educacional às suas respectivas estruturas de classificação originárias. Nesse sentido, há uma relação parte-todo que refere ao relacionamento entre um conceito ou entidade e suas partes constituintes [Keet, 2018]. Por exemplo a classe Estrutura_classificação (o todo), representa as taxonomias e esta necessita das instâncias da classe Objetivo_educacional (parte/componente), para representação e classificação dos níveis de aprendizagem das taxonomias formalizadas. Os indivíduos pertencentes às classes se relacionam através de propriedades do tipo ObjectProperties, que representam as relações presentes no domínio. Algumas destas propriedades foram definidas como: classificação, tem_objetivo, objetivo_atingido (transitiva), desempenho_atual, desempenho_atingido, tem_verbo e verbo_encontrado. A propriedade tem_objetivo mantém a relação entre indivíduos da classe Desempenho_academico e Objetivo_educacional. A propriedade desempenho_atual, estabelece a relação dos indivíduos da classe Estudante com indivíduos da classe Desempenho_academico. Através da propriedade tem_verbo é possível obter a

\footnotetext{
${ }^{1} \mathrm{http}: / /$ vowl.visualdataweb.org/protegevowl.html
} 
VII Congresso Brasileiro de Informática na Educação (CBIE 2018)

Anais do XXIX Simpósio Brasileiro de Informática na Educação (SBIE 2018)

informação sobre qual $\mathrm{OE}$ está relacionado com quais verbos e a propriedade verbo_encontrado informa quais os verbos obtidos a partir das interações dos diferentes alunos, indivíduos da classe Estudante, no SGA.

As Lógicas de Descrições (DL), do inglês Description Logic, são uma evolução dos formalismos de representação do conhecimento baseado em objeto (redes semânticas e frames), que correspondem a um subconjunto estruturado de lógica de primeira ordem [Vieira et al., 2005]. A sintaxe das DL é formada por símbolos representando conceitos (representação das classes) e papéis (propriedades dos conceitos), construtores (operadores que permitem a criação de conceitos complexos) e quantificadores (operadores que quantificam os papéis). A Tabela 1 apresenta as regras de DL desenvolvidas na ontologia proposta.

Tabela 1: Regras de lógica de descrição desenvolvidas

\begin{tabular}{|c|c|}
\hline Seq. & Regra de Lógica de descrição \\
\hline S1 & $\begin{array}{l}\left.\text { verbo_encontrado }(? \mathrm{p}, ? \mathrm{v}) \wedge{ }^{\wedge} \text { tem_verbo(?o, ?v }\right)^{\wedge} \text { tem_objetivo(?d, ?o) }->\text { desempenho_atual(?p, } \\
\text { ?d) }\end{array}$ \\
\hline $\mathrm{S} 2$ & classificacao(?o, ?c $)^{\wedge}$ tem_objetivo(?d, ?o) -> classificacao(?d, ?c) \\
\hline S3 & $\begin{array}{l}\text { desempenho_atual }(? \mathrm{p}, \quad ? \mathrm{~d} 1) \wedge \text { tem_objetivo(?d1, ?o1) } \wedge \text { objetivo_atingido(?o1, ?o2) } \wedge \\
\text { tem objetivo(?d2,?o2) }->\text { desempenho atingido(?p, ?d2) }\end{array}$ \\
\hline S4 & 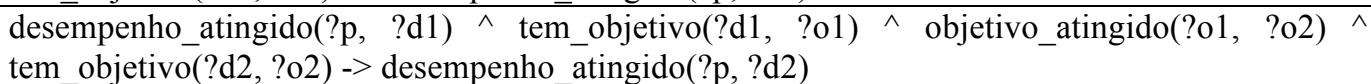 \\
\hline
\end{tabular}

As regras foram produzidas no padrão SWRL (Semantic Web Rule Language) e são expressadas em termos das classes, propriedades e indivíduos. A regra S1 tem por objetivo inferir sobre o desempenho atual do estudante tomando por base o verbo encontrado a partir da interação do aluno. A finalidade de S2 é definir em qual estrutura taxonômica (Bloom ou Bloom Revisada) um indivíduo da classe Desempenho_academico está representado. A regra S3 tem como objetivo inferir sobre o desempenho atingido a partir do desempenho atual do aluno, porém a taxonomia é uma estrutura de classificação hierárquica e, para atingir o próximo nível da estrutura, o aluno deve cumprir algum objetivo do nível anterior. A última regra, S4, tem como finalidade inferir sobre os níveis anteriores ao desempenho atual do aluno (desempenho_atingido), por exemplo: se um aluno estiver no nível 5 da taxonomia, ele deve (necessariamente) ter cumprido algum dos objetivos educacionais em cada nível anterior. Desta forma, a ontologia proposta possibilita a inferência do nível de aprendizagem do estudante relacionado a uma das Taxonomias (Bloom ou Revisada de Bloom) formalizadas nesta ontologia.

\section{Considerações Finais}

Esse trabalho em andamento apresenta um modelo ontológico que mantém o relacionamento das experiências de aprendizagem do aluno em SGA com os níveis de conhecimento da Taxonomia Revisada de Bloom. O objetivo é fornecer informações sobre o estado de conhecimento do aluno, possibilitando realizar a avaliação do desempenho acadêmico.

A promoção do desempenho acadêmico é fruto das interações dos alunos com o ambiente de ensino e, neste contexto, Learning Analytics surge como uma ferramenta para fins de compreensão e otimização do processo de ensino nos ambientes em que ocorre. Desta forma, as informações inferidas pela ontologia proposta farão parte de 
VII Congresso Brasileiro de Informática na Educação (CBIE 2018)

Anais do XXIX Simpósio Brasileiro de Informática na Educação (SBIE 2018)

uma arquitetura que possibilita auxiliar o educador na avaliação do desempenho dos estudantes, utilizando técnicas de Learning Analytics e Ontologias.

A contribuição deste trabalho é fornecer uma versão inicial de uma base de conhecimento, para pesquisadores da área de informática na educação, relacionada às taxonomias de Objetivos Educacionais mais referenciadas. Pretendemos, ainda, formalizar outras taxonomias e incluí-las nesta base de conhecimento. O próximo passo será a integração desta proposta com a arquitetura de um SGA. Assim, o educador poderá obter informações sobre o nível de aprendizagem do aluno realizando consultas nesta arquitetura.

\section{Referências}

Bloom, B. S.; Engelhart, M. D.; Furst, D. J.; Hill, W. H.; Krathwohl, D. R. (1956) Taxonomy of educational objectives: The classification of educational goals. NY: David McKay Company.

Costa, L. A.; Salvador, L. N.; Amorim, R. J. R. (2018) Evaluation of academic performance based on learning analytics and ontology: a systematic mapping study. IEEE Frontiers in Education. (no prelo).

Kalou, A; Solomou, G.; Pierrakeas C.; Kameas, A. (2012) An Ontology Model for Building, Classifying and Using Learning Outcomes. ICALT. DOI:10.1109/ICALT.2012.45.

Keet, C. Maria (2018) An Introduction to Ontology Engineering. Disponível em: https://people.cs.uct.ac.za/ mkeet/files/OEbook.pdf. Acessado em: agosto de 2018.

Lima, R. W.; Oliveira, A. M.; Silva, P. A.; Silva, Maria das Graças P. (2017) Ontologia para Gerenciamento de Objetos de Aprendizagem. SBIE. DOI: 10.5753/cbie.sbie.2017.1796.

Long, P.; Siemens, P.; George, L. (2011) Penetrating the Fog: Analytics in Learning and Education. In Educause Review, 46(5). Disponível em: https://er.educause.edu/ /media/files/article-downloads/erm1151.pdf\%20.

$\mathrm{Ng}$, Lai (2005) Ontological Model for Representation for Learning Objectives. Simon Fraser University. Tese (Simon Fraser University). 104f. Canadá. Disponível em: http://summit.sfu.ca/item/10336. Acesso em: abril de 2018.

Nussbaumer, A.; Scheffel, M.; Niemann, K.; Kravcik, M.; Albert, D. (2012) Detecting and reflecting learning activities in personal learning environments. Workshop on Awareness and Reflection in Technology-Enhanced Learning. Pp. 125-131. ISSN: 16130073.

Vieira, R.; Santos, D. A.; Silva, D. M.; Santana, M. R. (2005) Web semântica: ontologias, lógica de descrição e inferência. Tutorial do XI Simpósio Brasileiro de Sistemas Multimídia e Web (WEBMEDIA05).

Yago, H.; Clemente, J.; Rodriguez, D.; Fernandez-de-Cordoba, D. (2018) ONSMMILE: Ontology Network-based Student Model for MultIple Learning Environments, Data \& Knowledge Engineering. DOI: 10.1016/j.datak.2018.02.002. 